Article

\title{
Oral Bioavailability Evaluation of Celastrol-Encapsulated Silk Fibroin Nanoparticles Using an Optimized LC-MS/MS Method
}

\author{
Shuyu Zhan 1,2,*化, Amy Paik ${ }^{2}$, Felicia Onyeabor ${ }^{2}$, Baoyue Ding ${ }^{1,2}$, Sunil Prabhu ${ }^{2}$ and \\ Jeffrey Wang $2, *(\mathbb{D}$ \\ 1 Department of Pharmacy, College of Medicine, Jiaxing University, Jiaxing 314001, China; \\ lena_310@zjxu.edu.cn \\ 2 Department of Pharmaceutical Sciences, College of Pharmacy, Western University of Health Sciences, \\ Pomona, CA 91766, USA; amy.paik@westernu.edu (A.P.); felicia.onyeabor@westernu.edu (F.O.); \\ sprabhu@westernu.edu (S.P.) \\ * Correspondence: zhansy@zjxu.edu.cn (S.Z.); jwang@westernu.edu (J.W.)
}

Academic Editor: Yasumoto Nakazawa

Received: 3 June 2020; Accepted: 24 July 2020; Published: 28 July 2020

\begin{abstract}
Celastrol (CL), a compound isolated from Tripterygium wilfordii, possesses various bioactivities such as antitumor, anti-inflammatory and anti-obesity effects. In previous studies, we developed CL-encapsulated silk fibroin nanoparticles (CL-SFNP) with satisfactory formulation properties and in vitro cancer cytotoxicity effect. For further in vivo oral bioavailability evaluation, in this study, a simple and reliable LC-MS/MS method was optimized and validated to determine CL concentration in rat plasma. The separation of CL was performed on a C18 column (150 by $2 \mathrm{~mm}, 5 \mu \mathrm{m}$ ) following sample preparation using liquid-liquid extraction with the optimized extraction solvent of tert-butyl methylether. The assay exhibited a good linearity in the concentration range of $0.5-500 \mathrm{ng} / \mathrm{mL}$ with the lower limit of quantification (LLOQ) of $0.5 \mathrm{ng} / \mathrm{mL}$. The method was validated to meet the requirements for bioassay with accuracy of $91.1-110.0 \%$, precision $(\mathrm{RSD} \%$ ) less than $9.1 \%$, extraction recovery of $63.5-74.7 \%$ and matrix effect of $87.3-101.2 \%$. The developed method was successfully applied to the oral bioavailability evaluation of CL-SFNP. The pharmacokinetic results indicated the $\mathrm{AUC}_{0-\infty}$ values of $\mathrm{CL}$ were both significantly $(p<0.05)$ higher than those for pure CL after intravenous (IV) or oral (PO) administration of equivalent $\mathrm{CL}$ in rats. The oral absolute bioavailability $(F, \%)$ of CL significantly $(p<0.05)$ increased from $3.14 \%$ for pure CL to $7.56 \%$ for CL-SFNP after dosage normalization. This study provides valuable information for future CL product development.
\end{abstract}

Keywords: LC-MS/MS; celastrol; silk fibroin nanoparticles; pharmacokinetics; bioavailability

\section{Introduction}

Celastrol (CL, Figure 1A), a quinone methide triterpenoid isolated from Tripterygium wilfordii, possesses various bioactivities such as antitumor, anti-inflammatory and anti-obesity effects, and have been demonstrated to have treatment potential on multiple cancers [1], autoimmune diseases [2], chronic inflammation [3], cardiovascular diseases [4] and neurodegenerative diseases [5]. However, the poor aqueous solubility, low therapeutic index and systemic toxicity of CL limited its clinical applications $[3,6]$. To overcome CL's defect as a prospective product in clinic, novel drug delivery systems of CL based on nanotechnology, including nanofibers [7], liposomes [8,9], nanomicelles [10,11] and various kinds of nanoparticles [12-16] using single or synergistic delivery strategy, were designed and evaluated. The clinical application of CL is limited mainly due to its unsatisfactory in vivo pharmacokinetic as well as pharmacodynamic profiles. Recently, we developed a celastrol-encapsulated silk fibroin 
nanoparticle (CL-SFNP) using a desolvation method [17,18]. In vitro drug release studies of CL-SFNP revealed a slow and sustained release of drug at physiological $\mathrm{pH}(\mathrm{pH} 7.4)$ and rapid release at lysosomal $\mathrm{pH}(\mathrm{pH} 4.5)$, and the in vitro cytotoxicity study against two human pancreatic cancer cell lines (PANC-1 and Mia PaCa-2) demonstrated increased growth inhibition with the nanoparticle formulation compared to pure CL. Therefore, it is necessary to further study this formulation's in vivo properties for possible product development of CL.

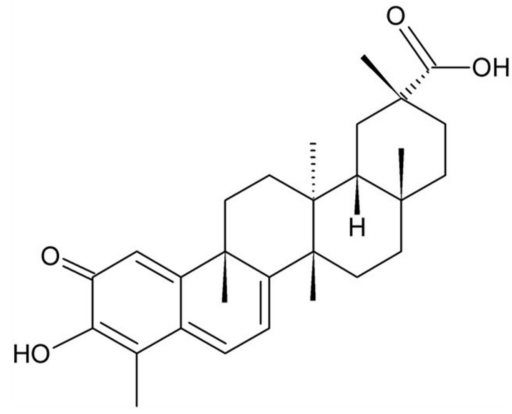

A

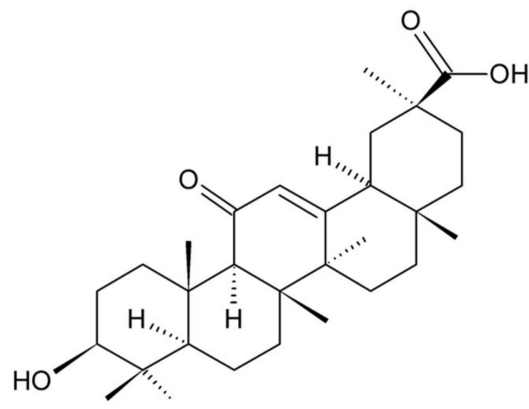

B

Figure 1. The chemical structure of celastrol (A) and 18- $\alpha$ glycyrrhetnic acid (IS) (B).

Pre-clinical pharmacokinetic evaluation including oral bioavailability is a vital part of the drug development process, and provides valuable information for the drug candidate's successful clinical translation. Nevertheless, the reliable determination methods are essential for the precise pharmacokinetics of the analyte and its novel formulations. Till now, some determination methods of CL in plasma based on HPLC technology and their application in pharmacokinetic studies have been reported [18-21]. However, the insufficient selectivity and sensitivity of HPLC methods apparently limit their wide application on in vivo pharmacokinetics such as oral bioavailability studies of CL loaded drug delivery systems. Zhang et al., reported an LC-MS/MS method for determination of $\mathrm{CL}$ in rat plasma, however, it needed complicated mobile phase, larger sample volume of $100 \mu \mathrm{L}$ and time-consuming sample preparation [22]. Likewise, no study has been reported to investigate CL's plasma sample preparation method concerning its high hydrophobic property leading to lower extraction recoveries of about $50 \%[18,22]$. Therefore, it is necessary to develop a simple and reliable determination method of CL in plasma with optimized sample preparation. In this study, in order to evaluate CL-SFNP's pre-clinical pharmacokinetics and bioavailability, we tried to develop a reliable LC-MS/MS method to determine CL in rat plasma coupled with simple and effective sample preparation.

\section{Materials and Methods}

\subsection{Chemicals, Reagents and Materials}

CL standard was purchased from Medkoo Biosciences Inc. (Morrisville, NC, USA). 18- $\alpha$ Glycyrrhetnic acid (Internal Standard, IS, Figure 1B) and HPLC-grade formic acid were bought from Sigma (St. Louis, MO, USA). Rat plasma was purchased from Innovative Research (Novi, MI, USA). HPLC-grade acetonitrile and methanol were purchased from VWR (Randor, PA, USA). Ultrapure water was produced by Millipore equipment (Millipore, Bedford, MA, USA). All other reagents were obtained from commercial sources and were of analytical grade.

\subsection{Chromatographic and Mass Spectrometry Conditions}

The LC-MS/MS system was composed of a SHIMADZU LC-20AD liquid chromatography system equipped with a SIL-20A auto sampler, and a triple-quadrupole tandem mass spectrometer (API 3200, Applied Biosystems, Foster City, CA, USA). The chromatographic separation was achieved on a 
Phenomenex Gemini C18 column (150 by $2 \mathrm{~mm}, 5 \mu \mathrm{m}$ ). The mobile phase consisted of $0.1 \%$ formic acid-2 $\mathrm{mM}$ ammonium acetate aqueous solution (A) and acetonitrile (B) using a gradient elution of $50 \% \mathrm{~B}$ at $0-2 \mathrm{~min}, 50-95 \% \mathrm{~B}$ at $2-3 \mathrm{~min}, 95 \% \mathrm{~B}$ at $3-6 \mathrm{~min}, 95-50 \% \mathrm{~B}$ at $6-7 \mathrm{~min}$ and the stop time was at $10 \mathrm{~min}$. The flow rate was $0.35 \mathrm{~mL} / \mathrm{min}$. The sample injection volume was $10 \mu \mathrm{L}$. CL and IS were both determined using positive ionization. The analytes were quantified under the multiple-reaction monitoring (MRM) mode. Mass spectrometry was operated with an optimized ion spray voltage at $3000 \mathrm{~V}$, turbo spray temperature at $500{ }^{\circ} \mathrm{C}$, and collision gas at 5 psi. The curtain gas, nebulizer gas (Gas 1) and auxiliary gas (Gas 2) were at 25, 40 and 20 psi, respectively. The precursor-to-product ion pair of $\mathrm{m} / \mathrm{z} 451.193 \rightarrow 201.096$ for $\mathrm{CL}$ and $\mathrm{m} / \mathrm{z} 471.293 \rightarrow 135.178$ for IS were monitored with the optimized declustering potential (DP), entrance potential (EP), collision energy (CE) and collision cell exit potential (CXP) of $31,7,33$ and $4 \mathrm{~V}$ and $81,12,33$ and $4 \mathrm{~V}$, respectively.

\subsection{Preparation of Calibration Standards and Quality Control Samples}

The stock solution of CL $(1.0000 \mathrm{mg} / \mathrm{mL})$ was prepared in methanol. A series of standard working solutions with the concentrations of 500, 200, 100, 50, 25, 5, 1 and $0.5 \mathrm{ng} / \mathrm{mL}$ were obtained by diluting the stock solution with methanol. The IS stock solution $(0.5 \mathrm{mg} / \mathrm{mL})$ was also prepared in the methanol. All solutions were stored at $4{ }^{\circ} \mathrm{C}$. To prepare calibration curves, each standard working solution $(50 \mu \mathrm{L})$ was evaporated to dryness in an Eppendorf Vacufuge Plus (Eppendorf, Germany). The residue was then spiked with $50 \mu \mathrm{L}$ blank rat plasma and mixed to form calibration standards. To validate the method, high, middle, and low quality control (QC) samples containing CL of 200, 25 and $1 \mathrm{ng} / \mathrm{mL}$, respectively, were prepared with the same treatment as the calibration standard samples. The calibration standard and QC samples were prepared for each analysis batch.

\subsection{Sample Preparation}

For CL analysis in rat plasma, the plasma samples were extracted using tert-butyl methylether based on liquid-liquid extraction technique. To each tube containing $50 \mu \mathrm{L}$ plasma, $1.0 \mathrm{~mL}$ tert-butyl methylether containing $1.0 \mu \mathrm{g} / \mathrm{mL}$ IS was added. The mixture was vortex-mixed for $5 \mathrm{~min}$ at $3000 \mathrm{rpm}$ using a VWR Pulsing Vortex Mixer (Randor, PA, USA) and centrifuged for $5 \mathrm{~min}$ at 10,000 rpm. Then, $900 \mu \mathrm{L}$ of supernatant was removed and placed into a new tube and evaporated at $30^{\circ} \mathrm{C}$ to dryness in the Eppendorf Vacufuge Plus (Eppendorf, Hamburg, Germany). The residue was reconstituted in $100 \mu \mathrm{L}$ methanol and centrifuged for $5 \mathrm{~min}$ at $10,000 \mathrm{rpm}$, and a $10.0 \mu \mathrm{L}$ aliquot was injected for the LC-MS/MS analysis within $18 \mathrm{~h}$.

\subsection{Method Validation}

\subsubsection{Specificity}

The specificity of LC-MS/MS method was evaluated by comparing the MRM chromatograms of blank rat plasma, blank rat plasma spiked with CL and IS, and rat plasmas collected at $0.5 \mathrm{~h}$ after drug administration. No observation of endogenous interference in the MRM chromatogram for CL and IS analysis was used to confirm the method's specificity.

\subsubsection{Linearity and Low Limit of Quantification}

The calibration curves were prepared by assaying standard plasma samples as described in preparation of calibration standards and quality control samples, and each level sample was prepared and assayed in duplicate in three separate days. The calibration curves were plotted by the peak area ratio (analyte/IS, y) versus the analyte concentration (x) using a $1 / \mathrm{x}^{2}$ weighted linear least-squares regression model. The lower limit of quantification (LLOQ) was defined as the lowest concentration on the calibration curve that produced a signal-to-noise $(\mathrm{S} / \mathrm{N})$ ratio of at least 10 with an acceptable accuracy (recovery within 80-120\%) and precision (RSD, below 20\%). 


\subsubsection{Precision and Accuracy}

The precision and accuracy were evaluated by analyzing six replicates of QC samples at low, medium and high concentration levels as described in preparation of calibration standards and quality control samples. The intra-day precision and accuracy of the assays were determined in the same day by analyzing six replicates at each concentration level. The inter-day precision and accuracy were evaluated on six consecutive days at each concentration level. The precision values expressed as RSD were required to be below $15 \%$, and the accuracy values expressed by recovery (mean measured concentration/spiked concentration $\times 100 \%$ ) were required to be within $85-115 \%$.

\subsubsection{Extraction Recovery and Matrix Effect}

The extraction recoveries were determined by comparing the peak area ratio (analyte/IS) from blank plasma samples spiked before extraction with those from blank plasma samples spiked after extraction at three levels of QC samples with IS $(n=6)$. The matrix effect at three levels of QC samples with IS $(n=6)$ was investigated by comparing the peak area ratios (analyte/IS) of CL and IS spiked with the blank plasma samples after extraction to those of pure standard solutions containing CL and IS at the same concentrations.

\subsubsection{Stability}

The stability of CL in plasma was assessed by analyzing six replicates which were spiked with standards at low, medium and high QC samples under different conditions: In autosampler after preparation for $18 \mathrm{~h}$, at $-20^{\circ} \mathrm{C}$ for 30 days and three freeze-thaw cycles. Newly prepared calibration curve was used for every stability test. The stability was evaluated by deviation, (spiked concentration-mean measured concentration)/(spiked concentration) $\times 100 \%$, which was required to be within $\pm 15 \%$.

\subsection{Pharmacokinetic Studies}

The study protocol (R18IACUC001) was approved by Western University of Health Sciences Institutional Animal Care and Use Committee (IACUC). Male Sprague-Dawley rats with pre-cannulated jugular vein were separated into four groups $(n=3)$. Two groups were used for intravenous (IV) administration study and the other two were used for oral (PO) administration study. The drug solutions of administration were prepared as pure CL solution in PEG 300 and CL-SFNP suspension in phosphate buffer solution (PBS), $\mathrm{pH} 7.4$ respectively. The rats were administered intravenously and intragastrically with the CL dose of $1 \mathrm{mg} / \mathrm{kg}$ and $3 \mathrm{mg} / \mathrm{kg}$, respectively. Two hundred $\mu \mathrm{L}$ of blood was collected respectively into pre-heparinized tubes at $0.083,0.5,1,2,4,6,8,12$ and $24 \mathrm{~h}$ after IV administration, and at $0.25,0.5,0.667,1,2,4,6,8,12$ and $24 \mathrm{~h}$ after PO administration. After $24 \mathrm{~h}$ the rats were euthanized with $30 \%$ isoflurane. Collected blood was then centrifuged for $15 \mathrm{~min}$ at 14,000 rpm. After centrifugation $110 \mu \mathrm{L}$ plasma was collected and transferred into an Eppendorf tube, and then stored at $-20^{\circ} \mathrm{C}$ until analysis. The IV samples were initially analyzed using an HPLC-UV method [18], but the low sensitivity of the method prohibited the analysis of the oral samples.

\subsection{Data Analysis}

Data were expressed as the mean \pm standard deviation (SD). The weighted linear least-squares regression model was performed using SPSS 16 software. The major pharmacokinetic parameters were estimated by non-compartmental analysis using WinNonlin 5.3 program package (Certara USA, Inc., Saint Louis, MO, USA). Differences between two groups were evaluated by an unpaired Student's $t$-test using Excel Statistical function (Microsoft, Redmond, WA, USA), and three groups were evaluated by one-way ANOVA using SPSS 16 software (IBM, Armonk, NY, USA). Differences were considered statistically significant at $p<0.05$. 


\section{Results and Discussion}

\subsection{LC-MS/MS Method}

In the LC-MS/MS method development, chromatographic condition was optimized, and the mobile phase with the additive of $0.1 \%$ formic acid- $2 \mathrm{mM}$ ammonium acetate in aqueous solution (A) and a gradient elution was chosen, which resulted in good separation and peak shapes of CL and IS. Owing to CL's high non-polarity, higher ratio of organic mobile phase (B) is usually needed to reduce elution time. However, it was found that high ratio $(90 \%)$ of organic mobile phase with an isocratic elution, causing CL's retention time at about $3 \mathrm{~min}$, could cause not only poor separation of CL from IS but also very high matrix effect (>200\%) from plasma especially for CL analysis at low concentrations. Therefore, to mediate the contradiction between elution time and separation as well as to reduce matrix effect, we applied a gradient elution of 50\% B at 0-2 min, 50-95\% B at 2-3 min, $95 \%$ B at 3-6 min, 95-50\% B at 6-7 min with the total analysis time of $10 \mathrm{~min}$, and injected the eluent into the MS system at 5.5 min using the valve as a diverter. As a result, the suitable chromatographic separation of CL and IS with the retention times of $7.31 \mathrm{~min}$ and $6.35 \mathrm{~min}$ respectively was obtained and the method exhibited no apparent matrix effect from plasma.

\subsection{Optimization of Sample Preparation}

Liquid-liquid extraction is the most popular plasma sample preparation technique because of its simple, convenient and reproducible procedure [23-26]. However, chaotic extraction recoveries of CL from plasma based on LLE have been reported, from about $60 \%$ to $90 \%$ using ethyl acetate as extract solvent with one-step extraction $[27,28]$, from about $50 \%$ to $90 \%$ using acetonitrile as extract solvent with one-step extraction $[18,29]$, and about 50\% using trichloromethane as extract solvent with two-step extraction [22]. In this study, we tried to optimize extract solvent to improve extraction recovery by investigating a wide-range of organic solvents of acetonitrile, methanol, ethyl acetate, 2-butanone, hexane, ethyl butrate, iso-propyl ether, tert-butyl methylether, dichloromethane and trichloromethane. To $50 \mu \mathrm{L}$ blank plasma sample spiked with $100 \mathrm{ng} / \mathrm{mL} \mathrm{CL}$ was added $1 \mathrm{~mL}$ extract solvent, and then was processed according to the method of sample preparation. Each solvent was investigated with three replicates. The peak area of CL obtained from each extract solvent is illustrated in Figure 2. It showed that tert-butyl methylether extracted the highest quantity of CL from plasma among all investigated solvents, so it was chosen as the extract solvent in our method. Using the optimized extract solvent, we could obtain extraction recovery of about $70 \%$ which was higher than some of those reported $[18,22]$. To test if increase of time and number of extractions would improve extraction efficiency, one-step extraction with $5 \mathrm{~min}$, one-step extraction with $10 \mathrm{~min}$ and two-step extraction with each $5 \mathrm{~min}$ were further investigated at three levels of QC samples with IS $(n=6)$. The peak area ratios (CL/IS) obtained from different extract conditions are listed in Table 1. It shows, for all three concentration levels of CL, no significant difference $(p>0.05)$ of peak area ratios existed among different extract conditions. Taken together, one-step extraction with 5 min was chosen as the simple and time-saving extraction method with recovery of about $70 \%$ in sample preparation. Incomplete extraction of CL from plasma might be due to its high hydrophobicity or partially irreversible plasma protein binding, which needs to be further investigated.

Table 1. The peak area ratios (celastrol (CL)/IS) obtained from investigation of different extract conditions for celastrol determination in rat plasma $(n=6)$.

\begin{tabular}{cccc}
\hline Concentration $(\mathbf{n g} / \mathbf{m L})$ & $\begin{array}{c}\text { One-Step Extraction } \\
\text { with } \mathbf{5} \text { min }\end{array}$ & $\begin{array}{c}\text { One-Step Extraction } \\
\text { with } \mathbf{1 0} \text { min }\end{array}$ & $\begin{array}{c}\text { Two-Step Extraction } \\
\text { with Each } \mathbf{5} \text { min }\end{array}$ \\
\hline 1 & $0.0035 \pm 0.0003$ & $0.0036 \pm 0.0002$ & $0.0037 \pm 0.0003$ \\
25 & $0.0881 \pm 0.0037$ & $0.0858 \pm 0.0041$ & $0.0899 \pm 0.0039$ \\
200 & $1.0483 \pm 0.1025$ & $0.9752 \pm 0.0604$ & $1.0361 \pm 0.0541$ \\
\hline
\end{tabular}




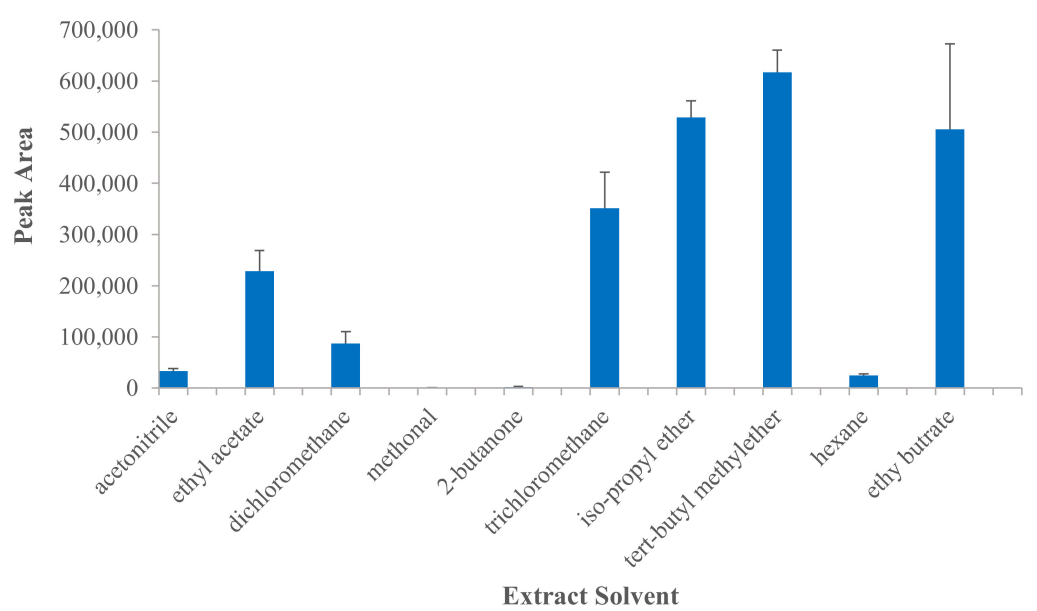

Figure 2. Peak area levels of $100 \mathrm{ng} / \mathrm{mL}$ celastrol in rat plasma using different extract solvents $(n=3)$.

\subsection{Method Validation}

\subsubsection{Specificity}

The representative chromatograms of a blank plasma sample, a blank plasma sample spiked with CL and IS, and two plasma samples obtained at $0.5 \mathrm{~h}$ after IV and PO administration of CL-SFNP suspension are shown in Figure 3. No significant interferences from endogenous substances in the blank plasma sample were observed at the retention times of CL and IS. Good specificity and selectivity were thus observed for the method.
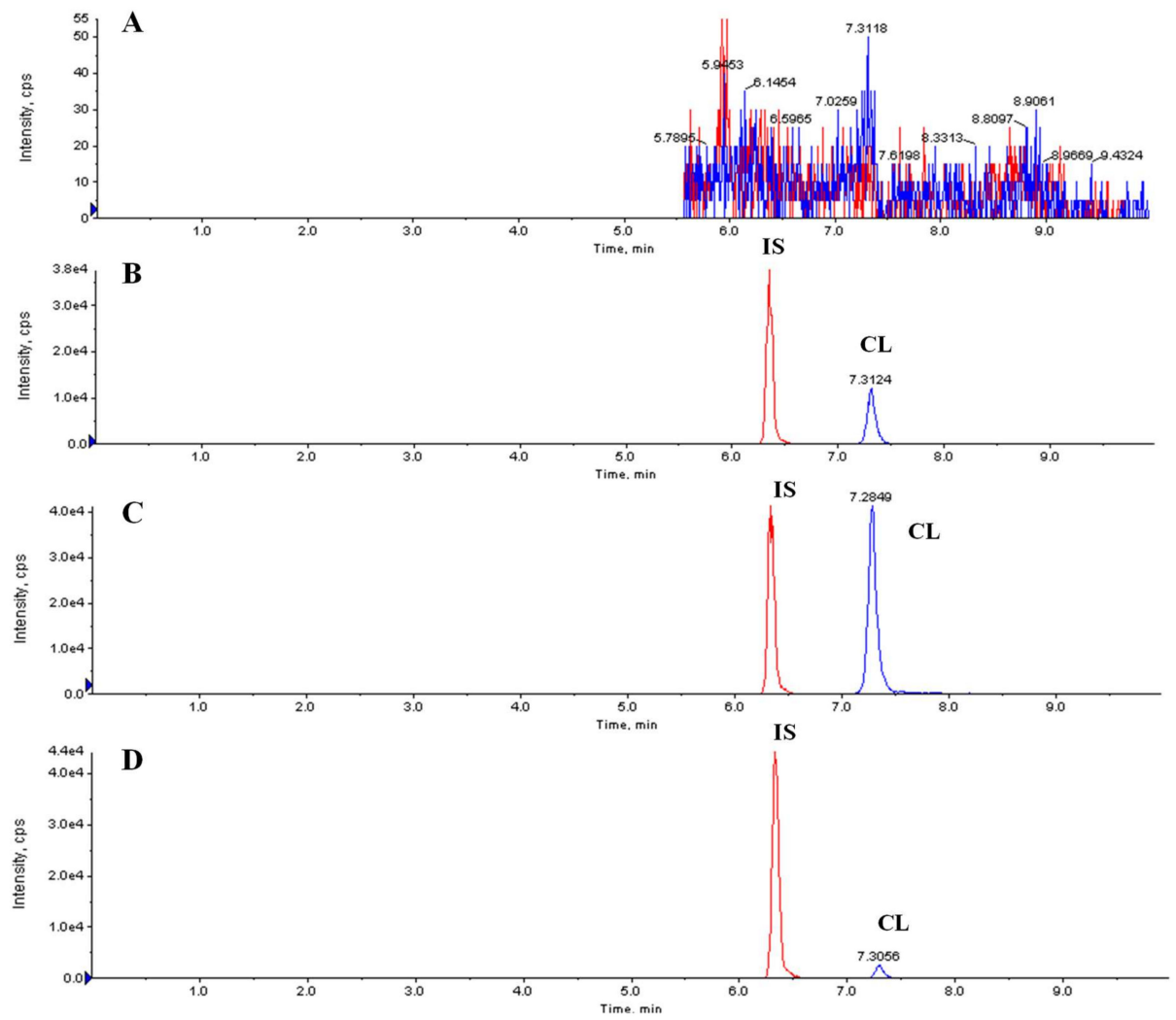

Figure 3. Representative multiple-reaction monitoring (MRM) chromatograms of CL and IS. (A) blank plasma; (B) blank plasma spiked with CL (100 ng/mL) and IS; (C) plasma sample obtained at $0.5 \mathrm{~h}$ after intravenous (IV) administration of celastrol-encapsulated silk fibroin nanoparticle (CL-SFNP) suspension; (D) plasma sample obtained at $0.5 \mathrm{~h}$ after oral (PO) administration of CL-SFNP suspension. 


\subsubsection{Linearity and LLOQ}

The linearity was validated in CL concentration range of $0.5-500 \mathrm{ng} / \mathrm{mL}$ by determining eight different concentration calibration standards in three separate days. Good linearity was obtained with a typical linear regression equation of $y=0.004 x\left(r^{2} \geq 0.976\right)$ using $1 / x^{2}$ weighting. LLOQ was $0.5 \mathrm{ng} / \mathrm{mL}$ with recovery of $91.1-112.5 \%$ and RSD of $11.2 \%$. Although the sensitivity with LLOQ of $0.5 \mathrm{ng} / \mathrm{mL}$ was a little lower than that reported $(0.1 \mathrm{ng} / \mathrm{mL})$ [22], it is meaningful for our method because we used a smaller plasma sample volume of $50 \mu \mathrm{L}$, which would add benefit for multiple time points pharmacokinetic studies.

\subsubsection{Precision and Accuracy}

The intra-day and inter-day precision and accuracy of the method were determined by replicating analytes of QC samples at 1, 25 and $200 \mathrm{ng} / \mathrm{mL}$ concentration levels. The results are listed in Table 2 . The mean accuracy of the analyte was within the range of $91.1-110.0 \%$. The intra- and inter-day precisions (RSD \%) of these analytes were less than $9.1 \%$ and $11.7 \%$, respectively. The results demonstrated the method developed in this study was precise and accurate.

Table 2. Precision and accuracy of the assay of celastrol in rat plasma $(n=6)$.

\begin{tabular}{ccccccc}
\hline \multirow{2}{*}{$\begin{array}{c}\text { Concentration } \\
\text { Spiked } \\
(\mathbf{n g} / \mathbf{m L})\end{array}$} & $\begin{array}{c}\text { Concentration } \\
\text { Measured (ng/mL) }\end{array}$ & $\begin{array}{c}\text { Accuracy } \\
\mathbf{( \% )}\end{array}$ & $\begin{array}{c}\text { Precision } \\
\mathbf{( \% )}\end{array}$ & $\begin{array}{c}\text { Concentration } \\
\text { Measured (ng/mL) }\end{array}$ & $\begin{array}{c}\text { Accuracy } \\
\mathbf{( \% )}\end{array}$ & $\begin{array}{c}\text { Precision } \\
(\%)\end{array}$ \\
\hline 1 & $0.91 \pm 0.08$ & 91.1 & 9.1 & $1.03 \pm 0.07$ & 102.6 & 7.2 \\
25 & $26.77 \pm 2.40$ & 107.1 & 9.0 & $24.38 \pm 2.85$ & 97.5 & 11.7 \\
200 & $220.03 \pm 13.15$ & 110.0 & 6.0 & $196.76 \pm 19.74$ & 98.4 & 10.0 \\
\hline
\end{tabular}

\subsubsection{Extraction Recovery and Matrix Effect}

The extraction recovery and matrix effect of $C L$ in rat plasma are shown in Table 3. It shows relatively high extraction recovery at three concentration levels with the mean values of $67.0 \%, 63.5 \%$ and $74.7 \%$. The matrix effects of $C L$ at three concentration levels were found to be within the range of 87.3-101.2\%, demonstrating no apparent influence of rat plasma matrix on CL determination.

Table 3. The extraction recovery and matrix effect of celastrol in rat plasma $(n=6)$.

\begin{tabular}{ccc}
\hline Concentration $(\mathbf{n g} / \mathbf{m L})$ & Extraction Recovery (\%) & Matrix Effect $(\%)$ \\
\hline 1 & $67.0 \pm 8.9$ & $101.2 \pm 8.8$ \\
25 & $63.5 \pm 2.5$ & $87.3 \pm 5.9$ \\
200 & $74.7 \pm 2.3$ & $98.0 \pm 11.0$ \\
\hline
\end{tabular}

\subsubsection{Stability}

Results from stability studies of CL are shown in Table 4. It demonstrates that CL in rat plasma were stable in storage at $-20^{\circ} \mathrm{C}$ for 30 days and after three cycles of freeze-thaw, and processed samples were stable for $18 \mathrm{~h}$ in autosampler. The stabilities of CL were acceptable at the above conditions and would satisfy the requirements of a routine pharmacokinetic study.

Table 4. The stability of celastrol in rat plasma $(n=6)$.

\begin{tabular}{|c|c|c|c|c|c|c|}
\hline \multirow{2}{*}{$\begin{array}{l}\text { Concentration } \\
\text { Spiked (ng/mL) }\end{array}$} & \multicolumn{2}{|c|}{$\begin{array}{l}\text { In Autosampler after } \\
\text { Preparation for } 18 \mathrm{~h}\end{array}$} & \multicolumn{2}{|c|}{ After Three Freeze-Thaw Cycles } & \multicolumn{2}{|c|}{ At $-20^{\circ} \mathrm{C}$ for 30 Days } \\
\hline & $\begin{array}{c}\text { Concentration } \\
\text { Measured (ng/mL) }\end{array}$ & $\begin{array}{l}\text { Deviation } \\
\quad(\%)\end{array}$ & $\begin{array}{c}\text { Concentration } \\
\text { Measured (ng/mL) }\end{array}$ & $\begin{array}{l}\text { Deviation } \\
(\%)\end{array}$ & $\begin{array}{c}\text { Concentration } \\
\text { Measured (ng/mL) }\end{array}$ & $\begin{array}{l}\text { Deviation } \\
\quad(\%)\end{array}$ \\
\hline 1 & $0.88 \pm 0.06$ & -12.4 & $0.95 \pm 0.09$ & -5.1 & $0.97 \pm 0.08$ & -3.3 \\
\hline 25 & $25.44 \pm 3.14$ & 1.8 & $24.28 \pm 1.82$ & -2.9 & $23.32 \pm 2.02$ & -6.7 \\
\hline
\end{tabular}




\subsection{Pharmacokinetic and Bioavailability Evaluation}

The validated method was used to investigate the pharmacokinetic profiles of CL in rats after single IV and PO administration of pure CL and CL-SFNP, respectively. Mean concentration-time curves of CL are showed in Figure 4. Meanwhile, the major pharmacokinetic parameters estimated by non-compartmental analysis are listed in Table 5; Table 6. For IV administration, it was found that $\mathrm{C}_{\max }$ and $\mathrm{AUC}_{0-\infty}$ of $\mathrm{CL}$ after CL-SFNP administration were $4414.8 \pm 1666.1 \mathrm{ng} / \mathrm{mL}$ and $8646.1 \pm 1998.9 \mathrm{~h} * \mathrm{ng} / \mathrm{mL}$ respectively, which were significantly $(p<0.05)$ higher than that after pure CL administration $\left(1701.3 \pm 170.7 \mathrm{ng} / \mathrm{mL}\right.$ and $4697.7 \pm 723.0 \mathrm{~h}^{*} \mathrm{ng} / \mathrm{mL}$, respectively). This implied that CL loaded in silk fibroin nanocapsulation definitely increased its residence time and slowed its elimination in vivo. Similarly, it exhibited significant pharmacokinetics improvement for $\mathrm{PO}$ administration. $\mathrm{C}_{\max }$ $(90.5 \pm 49.2 \mathrm{ng} / \mathrm{mL})$ and $\mathrm{AUC}_{0-\infty}(1065.5 \pm 494.6 \mathrm{~h}$ * $\mathrm{ng} / \mathrm{mL})$ after CL-SFNP administration were higher than those $(35.1 \pm 7.9 \mathrm{ng} / \mathrm{mL}$ and $441.9 \pm 82.6 \mathrm{~h} * \mathrm{ng} / \mathrm{mL}$, respectively) after pure CL administration. Therefore, CL loaded in silk fibroin nanocapsulation improved its absorption and systemic exposure in vivo. In addition, it was found there were rebound peaks approximately at $2 \mathrm{~h}$ in IV, at $2 \mathrm{~h}$ and $6 \mathrm{~h}$ in PO administration of CL-SFNP, possibly indicating gastric secretion-enteral reabsorption and enterohepaticre recycling may be involved in the pharmacokinetics of CL-SFNP, which needs further investigation. In addition, bioavailability calculation was performed through dosage normalization according to IV dose of pure CL. It was found CL's mean oral absolute bioavailability $(F, \%)$ after PO administration of CL-SFNP was $7.56 \%$, more than two times higher than that of $3.14 \%$ after PO administration of pure CL. Therefore, bioavailability combining other parameters demonstrated an improvement on pharmacokinetic behavior of CL-loaded silk fibroin nanoparticles.
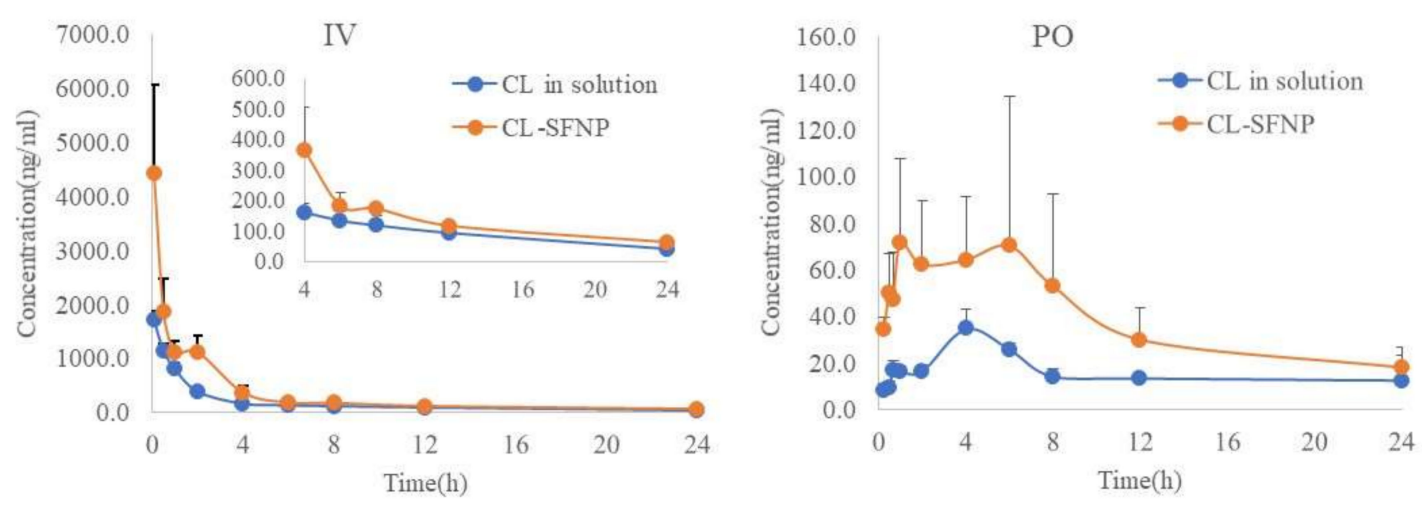

Figure 4. Mean concentration-time curves of CL after IV and PO administration of equivalent CL in pure CL and CL-SFNP ( $1 \mathrm{mg} / \mathrm{kg}$ and $3 \mathrm{mg} / \mathrm{kg}$, respectively) in rats $(n=3)$.

Table 5. Pharmacokinetics parameters of CL after intravenous administration of pure CL and CL-SFNP at $1 \mathrm{mg} / \mathrm{kg}(n=3)$.

\begin{tabular}{ccc}
\hline Parameters & CL in PEG 300 & CL-SFNP \\
\hline$k_{\mathrm{e} 1}\left(\mathrm{~h}^{-1}\right)$ & $0.0684 \pm 0.0092$ & $0.0640 \pm 0.0151$ \\
$T_{1 / 2 \beta}(\mathrm{h})$ & $10.27 \pm 1.47$ & $11.27 \pm 2.83$ \\
$C_{\max }(\mathrm{ng} / \mathrm{mL})$ & $1701.3 \pm 170.7$ & $4414.8 \pm 1666.1^{*}$ \\
$A U C_{0-\mathrm{t}}\left(\mathrm{h}^{*} \mathrm{ng} / \mathrm{mL}\right)$ & $4124.3 \pm 663.8$ & $7600.4 \pm 1658.8^{*}$ \\
$A U C_{0-\infty}\left(\mathrm{h}^{*} \mathrm{ng} / \mathrm{mL}\right)$ & $4697.7 \pm 723.0$ & $8646.1 \pm 1998.9^{*}$ \\
$V_{\mathrm{d}}(\mathrm{mL})$ & $990.3 \pm 272.1$ & $544.5 \pm 88.7$ \\
$C l(\mathrm{~mL} / \mathrm{h})$ & $66.3 \pm 12.5$ & $34.6 \pm 9.3 *$ \\
$M R T_{0-\infty}(\mathrm{h})$ & $9.47 \pm 0.77$ & $8.83 \pm 2.51$ \\
\hline
\end{tabular}


Table 6. Pharmacokinetics parameters of CL after oral administration of pure CL and CL-SFNP at $3 \mathrm{mg} / \mathrm{kg}(n=3)$.

\begin{tabular}{ccc}
\hline Parameters & CL in PEG 300 & CL-SFNP \\
\hline$T_{1 / 2}(\mathrm{~h})$ & $12.02 \pm 8.32$ & $8.97 \pm 2.57$ \\
$T_{\max }(\mathrm{h})$ & $4.67 \pm 1.15$ & $3.00 \pm 2.65$ \\
$C_{\max }(\mathrm{ng} / \mathrm{mL})$ & $35.1 \pm 7.9$ & $90.5 \pm 49.2$ \\
$A U C_{0-\mathrm{t}}\left(\mathrm{h}^{*} \mathrm{ng} / \mathrm{mL}\right)$ & $308.9 \pm 45.1$ & $842.9 \pm 567.9$ \\
$A U C_{0-\infty}(\mathrm{h} * \mathrm{ng} / \mathrm{mL})$ & $441.9 \pm 82.6$ & $1065.5 \pm 494.6^{*}$ \\
$V_{d}(\mathrm{~mL})$ & $367.1 \pm 279.0$ & $261.0 \pm 73.2$ \\
$C l(\mathrm{~mL} / \mathrm{h})$ & $20.5 \pm 1.4$ & $20.2 \pm 0.2$ \\
$M R T_{0-\infty}(\mathrm{h})$ & $17.01 \pm 8.95$ & $13.31 \pm 2.67$ \\
$F(\%)$ & $3.14 \pm 0.59$ & $7.56 \pm 3.51 *$ \\
\hline
\end{tabular}

Recently, improving the drugs' therapeutic efficiency using biopolymer nanoparticles has been a research focus. Silk fibroin is an excellent biopolymer of amphiphilic chemistry with the features of biocompatibility, biodegradablilty and low immunogenicity, and it can improve the bioavailability and pharmacokinetics of poor dissolution characteristic drugs [30]. Therefore, silk fibroin has been used to load many prospective anti-cancer entities with the purpose of cancer therapy improvement [31-33]. However, the in vivo pharmacokinetics investigation of drug loaded silk fibroin nanoparticles was relatively lacking. In this study, we performed pharmacokinetics and bioavailability evaluation of CL-SFNP by developing a reliable LC-MS/MS method of CL determination. The result of improved in vivo pharmacokinetic properties of CL-SFNP, combined with the favorable results from previous in vitro cytotoxicity study against cancer cells, gives hope for the potential of pharmacological activity in vivo of this formulation.

\section{Conclusions}

In conclusions, we developed a simple, sensitive, and reliable LC-MS/MS method to determine $\mathrm{CL}$ concentration in rat plasma. In the method, a simple one step liquid-liquid extraction technique using tert-butyl methylether as the extract solvent was applied with extraction recovery of about $70 \%$. The method exhibited higher sensitivity with the lower limit of quantification of $0.5 \mathrm{ng} / \mathrm{mL}$ using a small plasma volume of $50 \mu \mathrm{L}$, and meanwhile it demonstrated reliable accuracy, precision and stability. The developed method was successfully applied to the pharmacokinetic and bioavailability evaluation of CL after IV and PO administration of pure CL and CL-SFNP in rats. The results confirmed the improvement of $C L$ in vivo pharmacokinetics and oral bioavailability upon silk fibroin nanocapsulation. This study provides valuable information for future CL product development.

Author Contributions: S.Z.-Writing original draft, Methodology, and Funding acquisition; A.P., F.O. and B.D.-Investigation; S.P. and J.W.-Supervision, Manuscript revision \& editing, and Funding acquisition. All authors have read and agreed to the published version of the manuscript.

Funding: This research was partly funded by Natural Science Foundation of Zhejiang Province (LY18H280011) and National Natural Science Foundation of China (81503338).

Conflicts of Interest: The authors have declared no conflict of interest.

\section{References}

1. Kashyap, D.; Sharma, A.; Tuli, H.S.; Sak, K.; Mukherjee, T.; Bishayee, A. Molecular targets of celastrol in cancer: Recent trends and advancements. Crit. Rev. Oncol. Hematol. 2018, 128, 70-81. [CrossRef]

2. Venkatesha, S.H.; Moudgil, K.D. Celastrol suppresses experimental autoimmune encephalomyelitis via MAPK/SGK1-regulated mediators of autoimmune pathology. Inflamm. Res. 2019, 68, 285-296. [CrossRef] [PubMed] 
3. Cascao, R.; Fonseca, J.E.; Moita, L.F. Celastrol: A Spectrum of Treatment Opportunities in Chronic Diseases. Front. Med. 2017, 4, 69. [CrossRef] [PubMed]

4. Saito, K.; Davis, K.C.; Morgan, D.A.; Toth, B.A.; Jiang, J.; Singh, U.; Berglund, E.D.; Grobe, J.L.; Rahmouni, K.; Cui, H. Celastrol Reduces Obesity in MC4R Deficiency and Stimulates Sympathetic Nerve Activity Affecting Metabolic and Cardiovascular Functions. Diabetes 2019, 68, 1210-1220. [CrossRef] [PubMed]

5. Petrovic, A.; Kaur, J.; Tomljanovic, I.; Nistri, A.; Mladinic, M. Pharmacological induction of Heat Shock Protein 70 by celastrol protects motoneurons from excitotoxicity in rat spinal cord in vitro. Eur. J. Neurosci. 2019, 49, 215-231. [CrossRef]

6. Sanna, V.; Chamcheu, J.C.; Pala, N.; Mukhtar, H.; Sechi, M.; Siddiqui, I.A. Nanoencapsulation of natural triterpenoid celastrol for prostate cancer treatment. Int. J. Nanomed. 2015, 10, 6835-6846. [CrossRef] [PubMed]

7. $\quad$ Li, J.; Wang, X.; Jiang, H.; Lu, X.; Zhu, Y.; Chen, B. New strategy of photodynamic treatment of $\mathrm{TiO}_{2}$ nanofibers combined with celastrol for HepG2 proliferation in vitro. Nanoscale 2011, 3, 3115-3122. [CrossRef]

8. Soe, Z.C.; Thapa, R.K.; Ou, W.; Gautam, M.; Nguyen, H.T.; Jin, S.G.; Ku, S.K.; Oh, K.T.; Choi, H.G.; Yong, C.S.; et al. Folate receptor-mediated celastrol and irinotecan combination delivery using liposomes for effective chemotherapy. Colloids Surf. B Biointerfaces 2018, 170, 718-728. [CrossRef]

9. Qu, D.; Wang, L.; Qin, Y.; Guo, M.; Guo, J.; Huang, M.; Liu, Y.; Liu, C.; Li, H.; Chen, Y. Non-triggered sequential-release liposomes enhance anti-breast cancer efficacy of STS and celastrol-based microemulsion. Biomater. Sci. 2018, 6, 3284-3299. [CrossRef]

10. Li, P.; Zhou, X.; Qu, D.; Guo, M.; Fan, C.; Zhou, T.; Ling, Y. Preliminary study on fabrication, characterization and synergistic anti-lung cancer effects of self-assembled micelles of covalently conjugated celastrol-polyethylene glycol-ginsenoside Rh2. Drug Deliv. 2017, 24, 834-845. [CrossRef]

11. Zhao, Y.; Tan, Y.; Meng, T.; Liu, X.; Zhu, Y.; Hong, Y.; Yang, X.; Yuan, H.; Huang, X.; Hu, F. Simultaneous targeting therapy for lung metastasis and breast tumor by blocking the NF-kappaB signaling pathway using Celastrol-loaded micelles. Drug Deliv. 2018, 25, 341-352. [CrossRef] [PubMed]

12. Li, Z.; Yao, L.; Li, J.; Zhang, W.; Wu, X.; Liu, Y.; Lin, M.; Su, W.; Li, Y.; Liang, D. Celastrol nanoparticles inhibit corneal neovascularization induced by suturing in rats. Int. J. Nanomed. 2012, 7, 1163-1173.

13. Niemela, E.; Desai, D.; Nkizinkiko, Y.; Eriksson, J.E.; Rosenholm, J.M. Sugar-decorated mesoporous silica nanoparticles as delivery vehicles for the poorly soluble drug celastrol enables targeted induction of apoptosis in cancer cells. Eur. J. Pharm. Biopharm. 2015, 96, 11-21. [CrossRef] [PubMed]

14. Choi, J.Y.; Ramasamy, T.; Kim, S.Y.; Kim, J.; Ku, S.K.; Youn, Y.S.; Kim, J.R.; Jeong, J.H.; Choi, H.G.; Yong, C.S.; et al. PEGylated lipid bilayer-supported mesoporous silica nanoparticle composite for synergistic co-delivery of axitinib and celastrol in multi-targeted cancer therapy. Acta Biomater. 2016, 39, 94-105. [CrossRef] [PubMed]

15. Guo, L.; Luo, S.; Du, Z.; Zhou, M.; Li, P.; Fu, Y.; Sun, X.; Huang, Y.; Zhang, Z. Targeted delivery of celastrol to mesangial cells is effective against mesangioproliferative glomerulonephritis. Nat. Commun. 2017, 8, 1-17. [CrossRef]

16. Shan, W.G.; Wang, H.G.; Wu, R.; Zhan, Z.J.; Ma, L.F. Synthesis and anti-tumor activity study of water-soluble PEG-celastrol coupling derivatives as self-assembled nanoparticles. Bioorg. Med. Chem. Lett. 2019, 29, 685-687. [CrossRef] [PubMed]

17. Ding, B.; Wahid, M.A.; Wang, Z.; Xie, C.; Thakkar, A.; Prabhu, S.; Wang, J. Triptolide and celastrol loaded silk fibroin nanoparticles show synergistic effect against human pancreatic cancer cells. Nanoscale 2017, 9 , 11739-11753. [CrossRef] [PubMed]

18. Onyeabor, F.; Paik, A.; Kovvasu, S.; Ding, B.; Lin, J.; Wahid, M.A.; Prabhu, S.; Betageri, G.; Wang, J. Optimization of Preparation and Preclinical Pharmacokinetics of Celastrol-Encapsulated Silk Fibroin Nanoparticles in the Rat. Molecules 2019, 24, 3271. [CrossRef]

19. Peng, X.; Wang, J.; Song, H.; Cui, D.; Li, L.; Li, J.; Lin, L.; Zhou, J.; Liu, Y. Optimized preparation of celastrol-loaded polymeric nanomicelles using rotatable central composite design and response surface methodology. J. Biomed. Nanotechnol. 2012, 8, 491-499. [CrossRef]

20. Qi, X.; Qin, J.; Ma, N.; Chou, X.; Wu, Z. Solid self-microemulsifying dispersible tablets of celastrol: Formulation development, charaterization and bioavailability evaluation. Int. J. Pharm. 2014, 472, 40-47. [CrossRef]

21. Freag, M.S.; Saleh, W.M.; Abdallah, O.Y. Self-assembled phospholipid-based phytosomal nanocarriers as promising platforms for improving oral bioavailability of the anticancer celastrol. Int. J. Pharm. 2018, 535, 18-26. [CrossRef] 
22. Zhang, J.; Li, C.Y.; Xu, M.J.; Wu, T.; Chu, J.H.; Liu, S.J.; Ju, W.Z. Oral bioavailability and gender-related pharmacokinetics of celastrol following administration of pure celastrol and its related tablets in rats. J. Ethnopharmacol. 2012, 144, 195-200. [CrossRef] [PubMed]

23. Gangu Naidu, C.; Nageswara Rao, R.; Prasada Rao, A.V.; Nagesh Kumar, K.; Padiya, R.; Madhusudhan Rao, V. Supported liquid extraction and LC-MS-MS determination of iloperidone and olanzapine in rat plasma: Application to a pharmacokinetic study. J. Chromatogr. Sci. 2018, 56, 879-887. [CrossRef]

24. Xiao, J.; Wang, T.; Li, P.; Liu, R.; Li, Q.; Bi, K. Development of two step liquid-liquid extraction tandem UHPLC-MS/MS method for the simultaneous determination of Ginkgo flavonoids, terpene lactones and nimodipine in rat plasma: Application to the pharmacokinetic study of the combination of Ginkgo biloba dispersible tablets and Nimodipine tablets. J. Chromatogr. B Analyt. Technol. Biomed. Life Sci. 2016, 1028, 33-41. [PubMed]

25. Chandu, B.R.; Kanala, K.; Hwisa, N.T.; Katakam, P.; Khagga, M. Bioequivalance and pharmacokinetic study of febuxostat in human plasma by using LC-MS/MS with liquid liquid extraction method. Springerplus 2013, 2, 194. [CrossRef] [PubMed]

26. Bahrami, G.; Mohammadi, B. An isocratic high performance liquid chromatographic method for quantification of mycophenolic acid and its glucuronide metabolite in human serum using liquid-liquid extraction: Application to human pharmacokinetic studies. Clin. Chim. Acta 2006, 370, 185-190. [CrossRef]

27. Wang, W.; Liu, K.; Dong, H.; Liu, W. High-performance liquid chromatography spectrometric analysis of tripterin in rat plasma. J. Chromatogr. B Analyt. Technol. Biomed. Life Sci. 2008, 863, 163-166. [CrossRef]

28. Ouyang, X.K.; Cai, M.Q.; Chen, X.H.; Jin, M.C. Development and validation of a liquid chromatography coupled with atmospheric-pressure chemical ionization ion trap mass spectrometric method for the simultaneous determination of triptolide, tripdiolide, and tripterine in human serum. J. Anal. Toxicol. 2008, 32, 737-743. [CrossRef]

29. Wang, Z.; Chen, D.; Wang, Z. Effects of diclofenac on the pharmacokinetics of celastrol in rats and its transport. Pharm. Biol. 2018, 56, 269-274. [CrossRef]

30. Zhao, Z.; Li, Y.; Xie, M.B. Silk fibroin-based nanoparticles for drug delivery. Int. J. Mol. Sci. 2015, 16, 4880-4903. [CrossRef]

31. Fuster, M.G.; Carissimi, G.; Montalban, M.G.; Villora, G. Improving Anticancer Therapy with NaringeninLoaded Silk Fibroin Nanoparticles. Nanomaterials 2020, 10, 718. [CrossRef] [PubMed]

32. Perteghella, S.; Sottani, C.; Cocce, V.; Negri, S.; Cavicchini, L.; Alessandri, G.; Cottica, D.; Torre, M.L.; Grignani, E.; Pessina, A. Paclitaxel-Loaded Silk Fibroin Nanoparticles: Method Validation by UHPLC-MS/MS to Assess an Exogenous Approach to Load Cytotoxic Drugs. Pharmaceutics 2019, 11, 285. [CrossRef] [PubMed]

33. Montalban, M.G.; Coburn, J.M.; Lozano-Perez, A.A.; Cenis, J.L.; Villora, G.; Kaplan, D.L. Production of Curcumin-Loaded Silk Fibroin Nanoparticles for Cancer Therapy. Nanomaterials 2018, 8, 126. [CrossRef] [PubMed]

Sample Availability: Samples of the compound celastrol pure drug are available from the authors.

(C) 2020 by the authors. Licensee MDPI, Basel, Switzerland. This article is an open access article distributed under the terms and conditions of the Creative Commons Attribution (CC BY) license (http://creativecommons.org/licenses/by/4.0/). 